Post-print version of:

Publisher: Elsevier

Journal paper: Mechanical Systems and Signal Processing 2017, 88 1-8

Title: Bladed wheels damage detection through Non-Harmonic Fourier Analysis improved algorithm

Authors: P. Neri

Creative Commons Attribution Non-Commercial No Derivatives License

$$
\text { (c) } \frac{1}{\mathrm{BY}} \text { () }
$$

DOI Link: https://doi.org/10.1016/i.ymssp.2016.11.010 


\title{
Bladed wheels damage detection through Non-Harmonic Fourier Analysis improved algorithm
}

\author{
P. Neri ${ }^{\mathrm{a}, *}$ \\ ${ }^{a}$ University of Pisa. DICI - Department of Civil and Industrial Engineering. Largo L. Lazzarino 2, 56122 Pisa, Italy
}

\begin{abstract}
Recent papers introduced the Non-Harmonic Fourier Analysis for bladed wheels damage detection. This technique showed its potential in estimating the frequency of sinusoidal signals even when the acquisition time is short with respect to the vibration period, provided that some hypothesis are fulfilled. Anyway, previously proposed algorithms showed severe limitations in cracks detection at their early stage. The present paper proposes an improved algorithm which allows to detect a blade vibration frequency shift due to a crack whose size is really small compared to the blade width. Such a technique could be implemented for condition-based maintenance, allowing to use non-contact methods for vibration measurements. A stator-fixed laser sensor could monitor all the blades as they pass in front of the spot, giving precious information about the wheel health. This configuration determines an acquisition time for each blade which become shorter as the machine rotational speed increases. In this situation, traditional Discrete Fourier Transform analysis results in poor frequency resolution, being not suitable for small frequency shift detection. Non-Harmonic Fourier Analysis instead showed high reliability in vibration frequency estimation even with data samples collected in a short time range. A description of the improved algorithm is provided in the paper, along with a comparison with the previous ones. Finally, a validation of the method is presented, based on finite element simulations results.
\end{abstract}

Keywords: Bladed wheel resonance; Bladed wheels monitoring; Blade damage detection; Non-Harmonic Fourier Analysis.

${ }^{*}$ Corresponding author: Paolo Neri

Ph. +39 (0)50 2218019 Fax +39 (0)50 2210604

Email address: paolo.neri@dici.unipi.it (P. Neri) 


\section{Nomenclature}

NHFA Non-Harmonic Fourier Analysis.

DFT Discrete Fourier Transform.

LSF Least Square Fit.

$y(t) \quad$ Time varying signal.

$y_{i} \quad i-$ th sample of the time varying signal $y(t)$.

$n_{\mathrm{p}} \quad$ Number of samples of the acquired signal.

$\omega \quad$ Generic angular frequency for the NHFA calculation.

$H_{i, k} \quad i$ - th element of the $k-$ th sinusoidal component of the NHFA, computed at the angular frequency $\omega_{k}$.

$a(\omega) \quad$ Coefficient of the cosine term of the NHFA.

$C_{i}(\omega)$ Cosine term of the $i-$ th component of the NHFA.

$b(\omega) \quad$ Coefficient of the sine term of the NHFA.

$S_{i}(\omega) \quad$ Sine term of the $i-$ th component of the NHFA.

$F \quad$ Sample acquisition frequency.

$\tau \quad$ Sample time-length.

$y_{i}^{\prime} \quad$ Residual signal after first NHFA components subtraction.

$a^{\prime}(\omega) \quad$ Coefficient of the cosine term of the NHFA, computed on $y_{i}^{\prime}$.

$b^{\prime}(\omega) \quad$ Coefficient of the sine term of the NHFA, computed on $y_{i}^{\prime}$.

$R_{i, k} \quad$ Residual to be minimized when computing LSF at the angular frequency $\omega_{k}$.

$\Theta \quad$ Phase angle of the NHFA component.

$\omega_{\mathrm{r}} \quad$ Reference frequency used to compute NHFA during blade monitoring. 


\section{Introduction}

Rotating machinery are widely used in industry for several applications. The cyclic load produced by the machine rotation can cause structures vibrations. Compressors and turbines bladed wheels represent a typical example of this phenomenon. A significant vibrational response may produce noise amplification, efficiency loss and fretting. Furthermore, high cycle fatigue failures may occur. These phenomena represent a relevant economic loss for industry, since plant shut-downs are needed in case of blades failures. Literature shows that the most frequent impeller failure mechanism is represented by blades damage due to vibrations [1,2]. Several papers showed that the main load acting on the blades is determined by the non-uniform inlet or outlet pressure distribution produced by the stator vanes $[3,4]$. The combination between structures mode shapes and loading conditions may result in resonance phenomena, with serious consequences on the bladed wheels performances $[5,6,7]$. The aforementioned issues can be investigated through experimental modal analysis and harmonic response analysis $[8,9]$, and prevented at design stage by avoiding resonance excitation $[10,11]$. The wellknown Campbell diagram criterion surely represents the simpler approach [12], just considering load and modal frequency matching. Anyway, bladed wheels are characterized by a high density of natural modes, so that it is almost impossible to avoid any matching between load harmonics and natural modes. Thus, the Campbell approach results in too conservative predictions. A more realistic criterion is given by the Singh's Advanced Frequency Evaluation (SAFE) diagram. This approach also takes into account the matching between the mode shapes and the spatial load distribution. The theoretical and experimental validity of this method is well documented in literature $[13,14,15,16]$

The discussed methods are surely useful for bladed wheels design and optimization. Anyway, components monitoring is still essential to prevent failures. Programmed maintenance can be more efficient if coupled with online monitoring methods, in order to reduce plants shut-downs. Usually these methods are based on the detection of the natural frequency shift determined by the crack propagation. Contact measurement techniques present severe limitations if applied to rotating machinery, because of the severe machine working conditions and the telemetry systems needed for data transmission $[17,18]$. This problems can be solved by considering contactless measurement techniques. Digital Image Correlation methods have been applied to vibration measurements, but really expensive equipment is needed to achieve the desired resolution. Furthermore, even high-speed cameras still impose severe frequency limitations $[19,20]$.

Tip-timing techniques can be a valuable alternative, but several sensors may be needed to obtain a good representation and the simpler and cheaper sensors still have a low precision [21, 22, 23]. A single sensor technique was presented in [24] and applied to axial machinery. The sensor(s) could be placed radially close to the rotor, thus no interaction was generated with the operating fluid. In centrifugal compressors instead, both inlet and outlet edges interact with the fluid, hence any sensor positioning would impact the machine fluid dynamic. Laser Doppler Vibrometers (LDV) provide a high precision and sensitivity measurement tool, with no need to place the sensor close to the component. Tracking LDV are available to measure the vibrational response at any blade location during the rotation [25, 26]. Anyway, this technique requires that the chosen point is visible for all rotation angle, and no position feedback is available to check if the proper blade location is measured during the rotation. These issues can be solved by using Eulerian LDV: all the blades are measured by a single fixed sensor. This method was successfully applied in low speed measurements [27]: two flat cantilever beams $(12.7 \times 75 \times 1 \mathrm{~mm})$ were attached to a hub. A DC motor guaranteed a $90 \mathrm{rpm}$ rotational speed and a shaker provided the vibration excitation source. A similar set up was also presented in [28]. The short acquisition time surely represents a limitation for this technique. The measurement time available for each blade decreases as the rotational speed increases. This implies a short time sample, causing a poor frequency resolution for the traditional Fourier Analysis [29, 30, 31]. The frequency shifts due to crack propagation are obviously really small, thus a poor frequency resolution is not acceptable. This is the reason why Non-Harmonic Fourier Analysis (NHFA) was studied in [2, 32], where the method was firstly proposed for bladed wheels damage detection. NHFA allows to analyse really short time signals by means of a Least Square Fit (LSF), in order to find the actual frequency contained in time samples which are really short compared to the signal period. An analytical study of 
the method was provided in [33], where a post processing algorithm for frequency shift detection was proposed. The method was tested on experimental and numerical data, proving its potential in terms of trend detection but showing low accuracy in terms of small frequency shift detection. In the present paper a new post-processing algorithm, still based on NHFA, is proposed. A comparison with the previous method is also provided, and the performances of the new algorithm are tested on numerical data.

\section{NHFA analytical basis}

\subsection{NHFA formulation}

The NHFA formulation allows to detect an arbitrary number of sinusoidal contributions contained in the analysed signal. This is done by an iterative process, determining one sinusoidal contribution at each step. The main advantage of this technique with respect to conventional Fourier Analysis is that each sinusoidal contribution can be computed at any frequency, so that the frequency resolution of the method is arbitrary. In particular, the recorded time signal $y(t)$ can be approximated by a sinusoidal component $H(\omega)$ at the generic angular frequency $\omega$ [33], where the single sample $y_{i}$ is approximated by the corresponding value of $H_{i}(\omega)$ :

$$
\begin{aligned}
& H_{i}(\omega)=a(\omega) C_{i}(\omega)+b(\omega) S_{i}(\omega) \\
& C_{i}(\omega)=\cos \left(\left(i-\left(n_{\mathrm{p}}+1\right) / 2\right) \omega / F\right) \\
& S_{i}(\omega)=\sin \left(\left(i-\left(n_{\mathrm{p}}+1\right) / 2\right) \omega / F\right) \\
& a(\omega)=\frac{\sum_{i=1}^{n_{\mathrm{p}}} y_{i} C_{i}(\omega)}{\sum_{i=1}^{n_{\mathrm{p}}} C_{i}^{2}(\omega)} \\
& b(\omega)=\frac{\sum_{i=1}^{n_{\mathrm{p}}} y_{i} S_{i}(\omega)}{\sum_{i=1}^{n_{\mathrm{p}}} S_{i}^{2}(\omega)}
\end{aligned}
$$

where $F=\left(n_{\mathrm{p}}-1\right) / \tau$ represents the data sample rate, $n_{\mathrm{p}}$ is the number of samples, $\tau$ is the sample time length $\left(t_{1}=-\tau / 2, t_{n_{\mathrm{p}}}=\tau / 2\right)$ and $y_{i}$ is the $i-$ th sample of the acquired signal $y(t)$. If the actual frequency contributions contained in the acquired signal are not known, it is possible to find them by means of LSF. To do so, a frequency range and an arbitrary frequency resolution can be chosen, since the sinusoidal component $H_{i}(\omega)$ can be computed at any angular frequency. Then, the first harmonic component $H_{i, 1}=H_{i}\left(\omega_{1}\right)$ can be found by looking for the frequency $\omega_{1}$ which satisfies the condition:

$$
\sum_{i=1}^{n_{\mathrm{p}}}\left(y_{i}-H_{i, 1}\right)^{2}=\min _{\omega}\left(\sum_{i=1}^{n_{\mathrm{p}}}\left(y_{i}-H_{i}(\omega)\right)^{2}\right)
$$

A simple solution algorithm to perform the LSF of Eq.2 is reported in Section 2.2. Once $\omega_{1}$ is found, it is possible to compute the first contribution $H_{i, 1}$ by using Eq.1. Then, the generic $k$ - th contribution can be found by following the scheme:

$$
\begin{aligned}
& y_{i}^{\prime}=y_{i}-\sum_{q=1}^{k-1} H_{i, q} \\
& H_{i, k}=a^{\prime}\left(\omega_{k}\right) C_{i}\left(\omega_{k}\right)+b^{\prime}\left(\omega_{k}\right) S_{i}\left(\omega_{k}\right)
\end{aligned}
$$


In Eq.3, $H_{i, k}$ is computed by solving the aforementioned LSF on the signal $y_{i}^{\prime}$. It is worth noting that the various $\omega_{k}$ are not related, thus an arbitrary frequency resolution can be used to evaluate the various sinusoidal components $H_{i, k}$.

\subsection{Least square fit computation}

The key feature of NHFA consists in finding the sinusoidal components of the acquired signal by computing the least square fit of Eq.2. To compute the $k-$ th signal harmonic, a frequency resolution is chosen, and the function $H_{i, k}$ is computed for all the frequencies contained in the studied frequency range, with the chosen frequency resolution. Then, the residual $R_{i, s}=y_{i}-H_{i, k}$ can be defined, so that:

$$
\sum_{i=1}^{n_{\mathrm{p}}} R_{i, s}^{2}=\sum_{i=1}^{n_{\mathrm{p}}}\left(y_{i}-H_{i, k}\right)^{2}=\sum_{i=1}^{n_{\mathrm{p}}} y_{i}^{2}+\sum_{i=1}^{n_{\mathrm{p}}} H_{i, k}^{2}-2 \sum_{i=1}^{n_{\mathrm{p}}} y_{i} H_{i, k}
$$

Rearranging the third and the fourth of Eqs.1, it can be found that $a \sum_{i=1}^{n_{\mathrm{p}}} C_{i}^{2}=\sum_{i=1}^{n_{\mathrm{p}}} y_{i} C_{i}$ and $b \sum_{i=1}^{n_{\mathrm{p}}} S_{i}^{2}=$ $\sum_{i=1}^{n_{\mathrm{p}}} y_{i} S_{i}$. Since $a$ and $b$ do not depend on the summation index $i$, they can be inserted in the summations. Then, by multiplying these equations by $a$ and $b$ respectively, and by using the definitions of Eq.1, it is possible to state:

$$
\sum_{i=1}^{n_{\mathrm{p}}} y_{i} H_{i, k}=\sum_{i=1}^{n_{\mathrm{p}}} y_{i} a C_{i}+\sum_{i=1}^{n_{\mathrm{p}}} y_{i} b S_{i}=\sum_{i=1}^{n_{\mathrm{p}}} a^{2} C_{i}^{2}+\sum_{i=1}^{n_{\mathrm{p}}} b^{2} S_{i}^{2}
$$

It is worth noting that the term $\sum_{i=1}^{n_{\mathrm{p}}} H_{i, k}^{2}$ of Eq.4 is equal to $\sum_{i=1}^{n_{\mathrm{p}}} a^{2} C_{i}^{2}+b^{2} S_{i}^{2}+2 \sum_{i=1}^{n_{\mathrm{p}}} a C_{i} b S_{i}$. Considering the definitions of Eq.1, $S_{i}$ is an odd function, while $C_{i}$ is an even function, so that the product $S_{i} C_{i}$ still is an odd function. If the signal $y_{i}$ is acquired between $-\tau / 2$ and $\tau / 2$, it can be proved that $\sum_{i=1}^{n_{\mathrm{p}}} a C_{i} b S_{i}=0$, since the summation of the odd function $S_{i} C_{i}$ is performed in the interval $(-\tau / 2, \tau / 2)$. This consideration, along with Eq.5, leads to:

$$
\sum_{i=1}^{n_{\mathrm{p}}} y_{i} H_{i, k}=\sum_{i=1}^{n_{\mathrm{p}}} H_{i, k}^{2}
$$

Finally, combining Eq.4 and Eq.6, it follows:

$$
\sum_{i=1}^{n_{\mathrm{p}}} R_{i, s}^{2}=\sum_{i=1}^{n_{\mathrm{p}}} y_{i}^{2}-\sum_{i=1}^{n_{\mathrm{p}}} H_{i, k}^{2}
$$

Since $\sum_{i=1}^{n_{\mathrm{p}}} y_{i}^{2}>0$ and $\sum_{i=1}^{n_{\mathrm{p}}} H_{i, k}^{2}>0$, Eq.7 shows that the sum of $R_{i, s}^{2}$ reaches its minimum when the function $\sum_{i=1}^{n_{\mathrm{p}}} H_{i, k}^{2}$ reaches its maximum. In conclusion, it is possible to solve the LSF of Eq.2 by looking for the angular frequency $\omega_{k}$ which satisfies the equation:

$$
\sum_{i=1}^{n_{\mathrm{p}}} H_{i}^{2}\left(\omega_{k}\right)=\max _{\omega}\left(\sum_{i=1}^{n_{\mathrm{p}}} H_{i}^{2}(\omega)\right)
$$

Using Eq.8, the solution of the LSF of Eq.2 is straight forward. 


\subsection{Frequency estimation sensitivity to the acquisition time}

The effect of the length of the acquisition time $\tau$ with respect to the signal period $T$ was analysed through an example. A pure sinusoidal signal having a frequency of $2 \mathrm{~Hz}$ was preliminary processed through the aforementioned NHFA procedure with decreasing $\tau$. The frequency estimation error was lower than $0.1 \%$ even when $\tau$ was 0.002 times the signal period $T$. This analysis confirmed the capability of the NHFA method to detect pure signal frequency with high accuracy even when $\tau$ is really short compared to the signal period $T$. The analysis was then repeated adding a white noise contribution: the ratio between the white noise amplitude and the pure signal amplitude was set to 0.01 . The results of the analysis are reported in Tab.1, showing that the estimation error is still lower than $2 \%$ for a ratio $\tau / T$ of 0.08 .

\begin{tabular}{lcc}
\hline \hline$\tau(\mathrm{s})$ & $\tau / \mathrm{T}$ & Estimation err. $(\%)$ \\
\hline 0.1 & 0.2 & 0.33 \\
0.08 & 0.16 & 0.49 \\
0.06 & 0.12 & 0.81 \\
0.04 & 0.08 & 1.77 \\
0.02 & 0.04 & 6.86 \\
\hline \hline
\end{tabular}

Table 1: Frequency estimation error with decreasing acquisition time $\tau$.

\section{Damage detection algorithms description}

\subsection{Previous algorithm}

The application of the NHFA to bladed wheels damage detection is documented in literature only in $[2,31,32$, 33]. In these papers, one natural frequency of the studied component is considered as a reference frequency $\omega_{\mathrm{r}}$. When the crack propagates in the blade, its natural frequency gradually decreases, so that the harmonic component contained in the acquired time sample shows a frequency shift. In all the previous paper the LSF approach was not used. A different approach was followed instead, in order to speed up the computation in real time application. The parameter $\Theta$ was defined as:

$$
\tan (\Theta)=-b(\omega) / a(\omega)
$$

This parameter was computed at the chosen reference angular frequency $\omega_{\mathrm{r}}$ which corresponds to a natural frequency of the blade, e.g. the first bending mode. The algorithm proposed in [33] required that just one harmonic component was present in the processed signal. Thus, a moving average filter was applied to the measured signal in order to cut the high frequency components, having just the first bending mode frequency in the filtered signal. Under appropriate hypothesis, it was found that the parameter $\Theta$ linearly depends on the difference between the actual signal angular frequency and the chosen $\omega_{\mathrm{r}}$. Hence, it is possible to detect a frequency shift (and thus the crack propagation) by monitoring the parameter $\Theta$ over time. This method guarantees a really short computational time, since just the coefficients $a\left(\omega_{\mathrm{r}}\right)$ and $b\left(\omega_{\mathrm{r}}\right)$ needs to be computed at the reference angular frequency. On the other hand, it was found that the parameter $\Theta$ also depends on several variables of the acquired signal, so that some relevant oscillations of the parameter were found to act as a noise source. These oscillations were reduced by applying a further moving average filter on the estimated frequency, improving the method efficiency. Anyway, since small crack propagation causes really small frequency shifts, it was not possible to detect the crack at its early stage. Fig.1(a) shows the flux diagram of the main steps of the algorithm proposed in [33]. In the example shown below, the described method was applied to a sample blade having $30 \mathrm{~mm}$ width. As shown in Fig.4, the estimated vibration frequency was subject to high oscillations which hid the smaller frequency variations. Specifically, the variation of the vibration frequency became higher 
than the typical oscillation amplitude when the crack reached about $10 \mathrm{~mm}$ length, which represents a huge dimension with respect to the total width of the blade. In conclusion, the method showed to be able to properly catch the trend of the blade vibration angular frequency as the crack grows, proving the potential of NHFA for damage detection through short time acquisitions. Anyway the accuracy level was not sufficient to provide a reliable tool for small cracks detection.

\subsection{Improved algorithm}

Previous works showed that NHFA approach is able to detect frequency shifts in short time acquired signals. Anyway severe limitations arose because of the oscillation of the monitored parameter $\Theta$. In order to overcome this issue, an improved algorithm was developed. A LSF approach was followed, in order to exactly detect the dominant frequency component of the measured signal. This means that the measured signal can be directly processed by the NHFA algorithm, which act as a filter itself. Thus, no preliminary filtering was needed, at least in principle. A really fine frequency resolution was needed, since really small frequency shifts need to be detected to achieve a early stage crack detection. In order to speed up the computation, the searching angular frequency range was reduced: a reference angular frequency $\omega_{\mathrm{r}}$ was chosen (e.g. the first bending mode) and only the sharp window $\omega_{\mathrm{r}}(1 \pm 0.1)$ was considered. This allowed to precisely detect the actual frequency contribution contained in the signal. The signal was firstly analysed without any filtering, since the NHFA method itself acts as a filter if the LSF is performed. Short time signal were considered, having an acquisition time which was about the double of the signal period. The proposed approach was tested on frequency varying signals, providing a reliable estimation of the signal frequency over time and a relevant improvement of the results with respect to the previous method (see Section 4.1). Anyway, some oscillations and uncertainties could be noted for the smaller frequency variations. This uncertainty could be further reduced by applying a moving average filter to the measured signal, right before the NHFA algorithm was used, as suggested in [33]. The moving average filter was chosen because it showed to provide better performances with respect to a Butterworth filter in this application [33]. A results comparison allowed to evaluate the beneficial effect of the filtering in terms of frequency estimation accuracy. The final flux diagram of the improved algorithm is reported in Fig.1(b).

\section{Method application}

\subsection{Algorithms comparison}

This improved algorithm was tested on virtual measurements obtained by the Finite Element (FE) analysis of a schematic rectangular blade $(30 \times 100 \times 0.5 \mathrm{~mm})$. Fixed boundary conditions were imposed to one end of the blade. The crack propagation was simulated by gradually removing elements in the chosen cut region. A preliminary modal analysis was performed in order to determine the expected natural frequencies corresponding to each crack size. The natural frequencies of the undamaged blade were found to be $42.35 \mathrm{~Hz}$ and $291.61 \mathrm{~Hz}$ for the first two modes, which were a bending mode and a torsional mode respectively. A transient analysis was finally performed by applying an impulsive load at the blade tip. The force was applied to a blade corner in order to excite both bending and torsional modes of the component, so that at least two frequency contributions were expected in the measured signal. No dumping was added in the model, thus the load could be applied in the first load step only. The transient analysis was divided in load steps of $0.4 \mathrm{~s}$, and the damage was increased of 1 $\mathrm{mm}$ at the beginning of each load step. Hence, a jump occurred in the actual signal frequency at the beginning of each load step, corresponding to the crack size increase. Fig.2 shows a schematic view of the developed FE model. The method was developed to be applied to Eulerian measurements, so the virtual measured signal was reconstructed by combining the displacement of the nodes belonging to the measurement line, as done in [33]: a sampling frequency and a fictitious blade rotational speed were chosen, thus it was possible to know which node was virtually pointed by the laser spot at each time instant. Hence, it was possible to determine that the $j$ - th node was pointed by the laser at the time instant $t_{j}$. The displacement of the $j$ - th node at the time $t_{j}$ was assumed to be the value of the virtual acquired signal corresponding to the instant $t_{j}$. This was repeated for each 


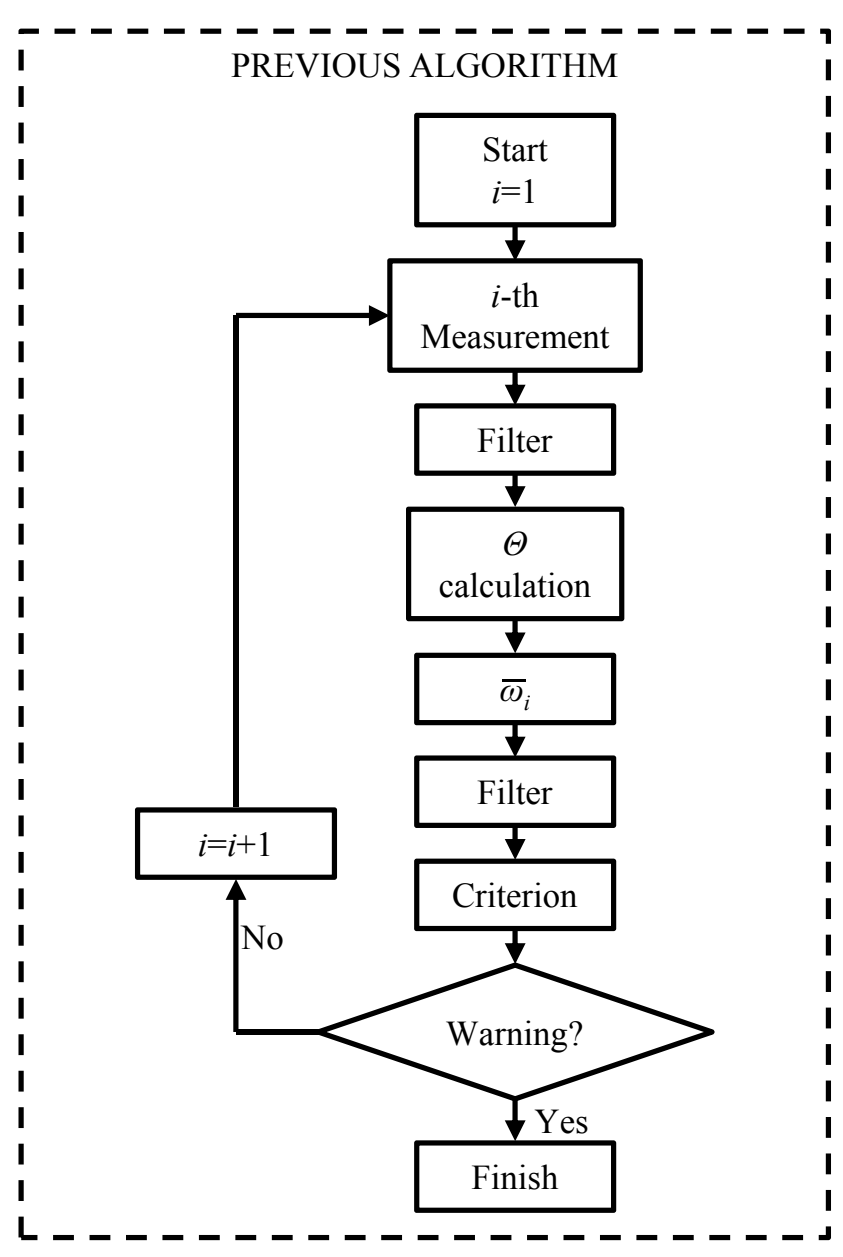

(a)

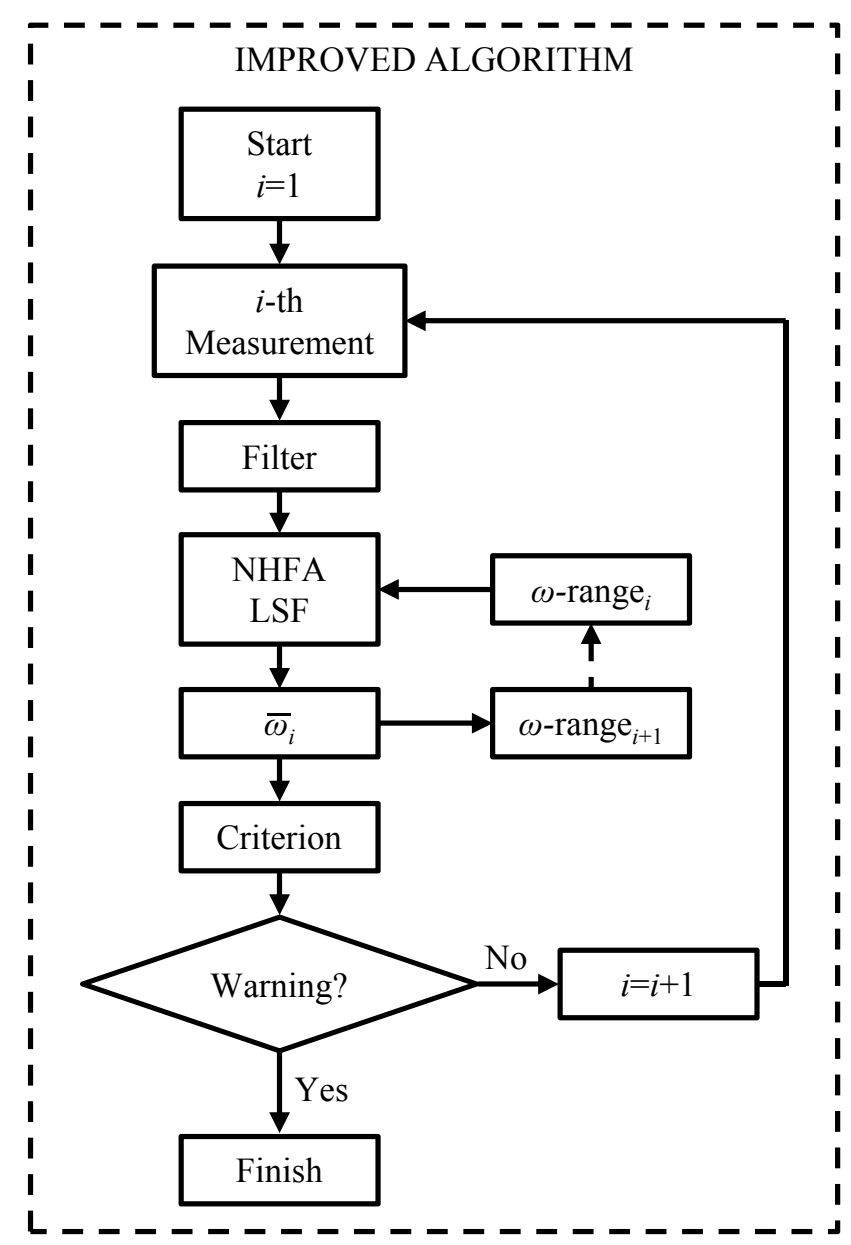

(b)

Figure 1: Flux diagram of the NHFA algorithms for damage detection: (a) previous algorithm and (b) improved LSF algorithm.

considered time instant $t_{q}$ and corresponding $q$ - th node, to create the simulated virtual measurement sample. Finally, a white noise was added to the virtual signal, to reproduce measurement noise. The ratio between signal amplitude and noise amplitude was set to 0.01 .

The first bending frequency was chosen for the analysis. This allowed to reduce the simulation time, since higher frequency phenomena would need a greater number of shorter time steps in the transient analysis. Anyway, the method could be applied at any natural mode, as long as the measurement sampling frequency is higher than twice the chosen natural frequency. This example was firstly used to investigate the effect of the moving average filter on the results of the improved algorithm. Fig.3(a) shows a comparison between the actual frequency of the considered signal (grey line) and the estimated one (cross marks) in the case of no filter applied to the measurement. The horizontal axis represents the simulation time: indeed the grey line (actual frequency) jumps each 0.4 $\mathrm{s}$, corresponding to the cut increase. The smaller frequency shifts corresponding to smaller crack sizes (so the firsts simulation load steps) were not remarkable with respect to the estimation uncertainty. This is due to the presence of the torsional frequency contribution and of the white noise in the acquired signal, which worsen the estimation accuracy. On the other hand, Fig.3(b) shows the results obtained with the improved algorithm when the moving average filter was applied to the processed signal. The plot shows a great improvement of the results, providing a reliable frequency estimation over time. In the proposed example, the considered acquisition time was $\tau=0.05 \mathrm{~s}\left(n_{\mathrm{p}}=51\right)$, which is about twice the first bending mode period. It is worth noting that a rough estimation of the natural frequencies of the blade is needed to properly set up the moving average filter. This 


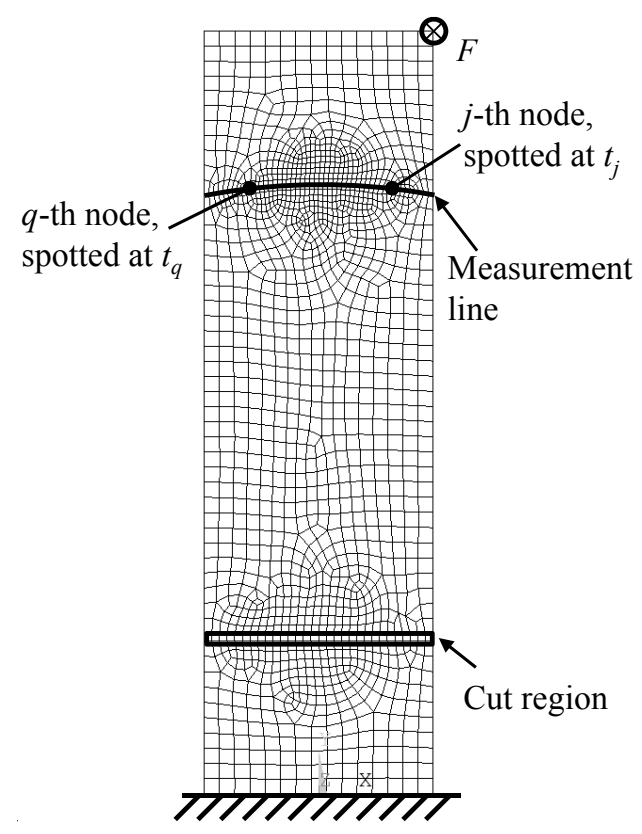

Figure 2: Finite element model scheme: blade with a growing crack.

estimation can be obtained by dedicated FE or experimental modal analysis. Otherwise, the frequencies can be estimated by the NHFA method itself if the undamaged blade vibration is preliminarily acquired and processed without filtering using Eqs.3.

Finally, a comparison between the previous algorithm and the improved one was performed by processing the same data with the two algorithms. Results obtained with the previous method are reported in Fig.4. The comparison between Fig.4 (previous method) and Fig.3(b) (improved method) shows that the improved algorithm achieved better performances with respect to the previous one, since the estimation uncertainty resulted drastically reduced.

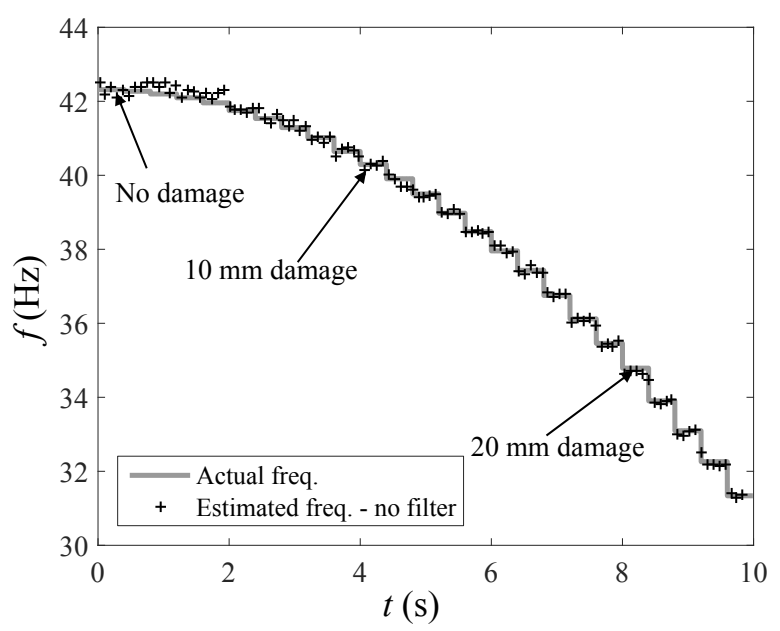

(a)

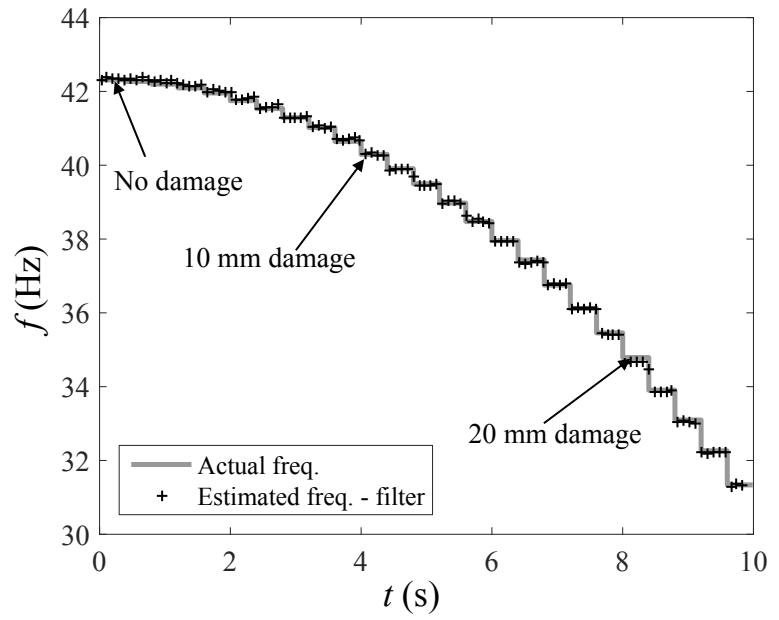

(b)

Figure 3: Improved algorithm results: (a) no filtering applied to the signal and (b) moving average filter applied to the signal. 


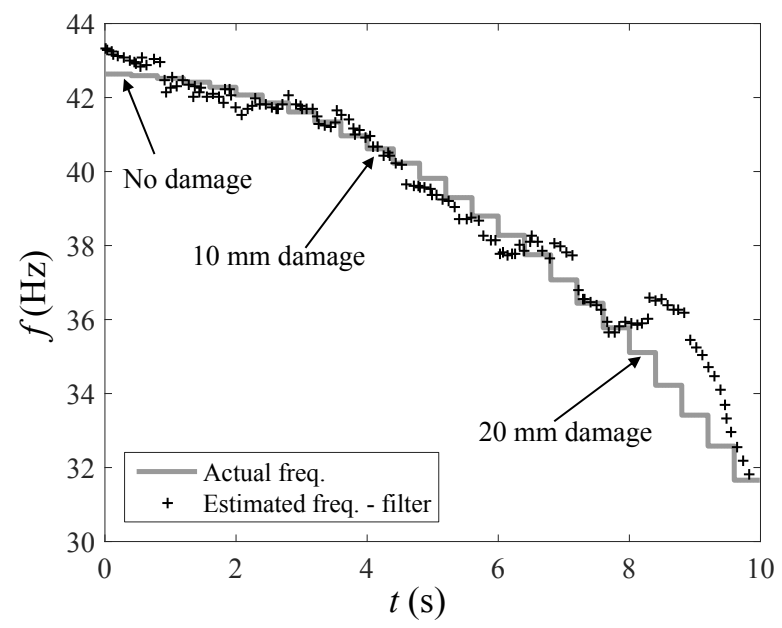

Figure 4: Frequency estimation with the previous algorithm for comparison with Fig.3(b).

\subsection{Damage detection}

The proposed method is able to estimate the blades vibration frequency as they pass in front of a sensor, even if the acquisition time is short with respect to the vibration period. Early stage cracks determine of course a small frequency shift, so that a high fidelity is needed in blades vibration frequency estimation. Fig.5 shows a zoomed view of the plot of Fig.3(b). The dashed line represents the estimated frequency as described in previous paragraph. If an oscillation range is chosen around the undamaged frequency of $42.35 \mathrm{~Hz}$ (e.g. \pm 0.07 was chosen for the grey line in Fig.5), it is possible to note that a $2 \mathrm{~mm}$ crack causes a warning message, while a $3 \mathrm{~mm}$ crack or greater is clearly detected by the method. On the other hand, the black line in Fig.5 represents the detected frequency when a further moving average filter is applied to the grey dashed line. This further data elaboration allows to reduce the oscillation range to \pm 0.03 , so that a $2 \mathrm{~mm}$ crack can already be detected using the proposed method.

\section{Conclusions}

Previous works about Non-Harmonic Fourier Analysis (NHFA) showed its potential in detecting frequency shifts in time varying signals, even if the acquisition time is short compared to the signal period. Anyway, existing algorithms are based on the computation of a parameter which is fluctuating mainly because of filtering issues. Because of these fluctuations, the method was only able to catch the frequency trend over time, but did not allow to detect cracks at their early stage.

An improved algorithm is then proposed in the present paper. The method is based on a Least Square Fit (LSF) procedure, which directly detects the main sinusoidal component of the signal. In this way, filtering issues are drastically reduced, since NHFA acts as a filter itself in the proposed approach. The improved algorithm was validated through Finite Element (FE) simulation data, showing a significant performance enhancement with respect to the previous algorithm. In particular, a crack propagation was simulated on a $30 \mathrm{~mm}$ rectangular blade. The crack was detected using the improved algorithm when the damage size reached $2 \mathrm{~mm}$. This test showed that NHFA can be successfully applied to early stage cracks detection if the LSF approach is adopted. It is worth noting that the presented method can only indicate that a degradation process is evolving in the blade, but it cannot be directly used to determine location and size of the crack in the blade.

The NHFA improved algorithm showed really good performances when used to estimate the vibration frequency of FE simulated data. Experimental analysis should be performed to asses the method effectiveness when applied to actual measurements. 


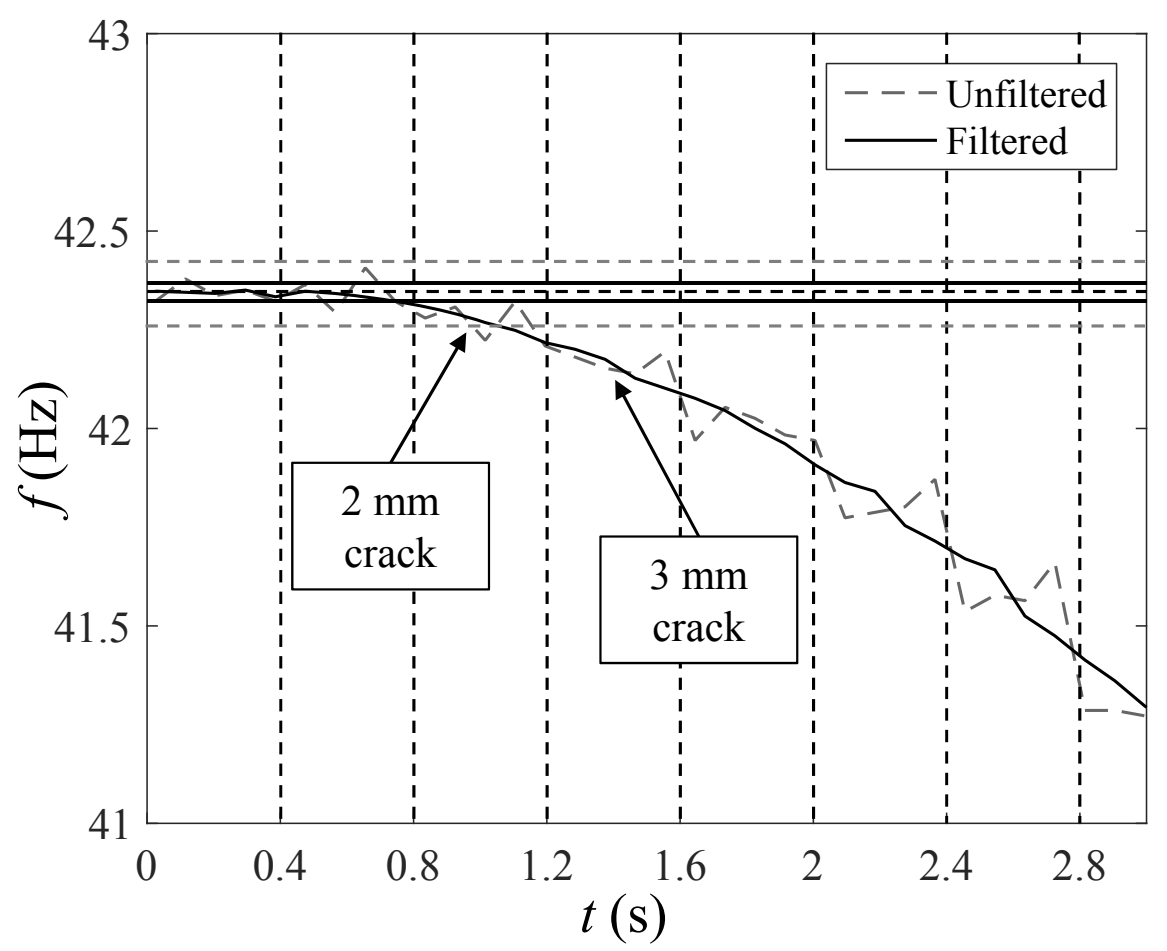

Figure 5: Estimated frequency using the improved algorithm: zoomed view for early stage damage detection.

\section{References}

[1] N. Rieger, The high cost of failure of rotating equipment., in: Proceedings of the 44th Meeting of the Mechanical Failures Prevention Group, 1990, pp. 1-17, aD-A226 670.

[2] A. Oberholster, P. Heyns, Online condition monitoring of axial-flow turbomachinery blades using rotor-axial eulerian laser doppler vibrometry, Mechanical Systems and Signal Processing 23 (5) (2009) 1634-1643, doi: 10.1016/j.ymssp.2009.01.001.

[3] A. Kammerer, R. Abhari, Experimental Study on Impeller Blade Vibration During Resonance - Part I: Blade Vibration Due to Inlet Flow Distortion, Journal of Engineering for Gas Turbines and Power 131 (2) (2009) art. no. 022508, doi: 10.1115/1.2968869.

[4] D. Raber, C. Williams, C. Hah, Inlet flow distortion and unsteady blade response in a transonic axial-compressor rotor, Tech. Rep. No. ISABE-99-7287, National Aeronautics and Space Administration. Glenn Research Center, Cleveland OH (1999).

[5] G. Nordwall, M. Leduc, A. Demeulenaere, Unsteady blade and disk resonant stress analysis due to supersonic inlet guide vane wakes, in: Proceedings of GT2008 ASME Turbo Expo 2008: Power for Land, Sea and Air, ASME, 2008, pp. 1-10, gT2008-50865, June 9-13, Berlin, Germany.

[6] M. Singh, T. Matthews, C. Ramsey, Fatigue Damage of Steam Turbine Blade Caused by Frequency Shift Due to Solid Buildup A Case Study, in: Proceedings of the 23rd Turbomachinery Symposium, 1994, pp. 107-114.

[7] R. Fleeting, R. Coats, Blade Failures in the H.P. Turbines of R.M.S. Queen Elizabeth 2 and their rectification, Institute of Marine Engineers Transactions 82 (2) (1970) 49-74.

[8] L. Bertini, P. Neri, C. Santus, Design and optimization of a compact high-frequency electromagnetic shaker, in: Proceedings of 11th International Conference on Engineering Vibration, 2015, pp. 1-9.

[9] P. Neri, Excitation device for high frequency vibration analysis: design and test results, Experimental Techniques. Submitted.

[10] N. Riever, Progress with the solution of vibration problems of steam turbine blades, Tech. rep., STI Technologies, Inc. (2003).

[11] N. Mukhopadhyay, S. G. Chowdhury, G. Das, I. Chattoraj, S. Das, D. Bhattacharya, An investigation of the failure of low pressure steam turbine blades, Engineering Failure Analysis 5 (3) (1998) 181-193, doi: 10.1016/S1350-6307(98)00016-8.

[12] R. Bishop, D. Johnson, The Mechanics of Vibration, Cambridge University Press, 1979.

[13] H. Bloch, M. Singh, Steam Turbines: design, applications and re-rating, 2nd Edition, McGraw-Hill, 2008.

[14] L. Bertini, P. Neri, C. Santus, A. Guglielmo, G. Mariotti, Analytical investigation of the safe diagram for bladed wheels, numerical and experimental validation, Journal of Sound and Vibration 333 (2014) 4771-4788. doi:10.1016/j.jsv.2014.04.061.

[15] L. Bertini, B. Monelli, P. Neri, C. Santus, A. Guglielmo, Robot assisted modal analysis on a stationary bladed wheel, in: Proceedings of the 12th Biennial Conference on Engineering Systems Design and Analysis (ESDA), 2014, pp. 1-8. doi:10.1115/ESDA2014-20636.

[16] L. Bertini, P. Neri, C. Santus, A. Guglielmo, Automated experimental modal analysis of bladed wheels with an anthropomorphic robotic station, Experimental Mechanics. Submitted. 
[17] S. Houde, R. Fraser, G. Ciocan, C. Deschênes, Part 1 - experimental study of the pressure fluctuations on propeller turbine runner blades during steady-state operation, in: Proceedings of the 26th IAHR Symposium on Hydraulic Machinery and Systems, 2012, pp. 1-14. doi:10.1088/1755.

[18] M. Sunar, B. Al-Bedoor, Vibration measurement of rotating blades using a root embedded pzt sensor, Shock and Vibration 15 (2008) 517-541. doi:10.1155/2008/494639.

[19] M. Helfrick, P. Pingle, C. Niezrecki, P. Avitabile, Optical non-contacting vibration measurement of rotating turbine blades, in: Proceedings of the IMAC-XXVII, 2009, pp. 1-5.

[20] J. Baqersad, T. Lundstrom, C. Niezrecki, P. Avitabile, Monitoring the dynamics of an operating helicopter rotor using 3d digital stereophotogrammetry, in: Proceedings of the 11th International Conference on Recent Advances in Structural Dynamics (RASD), 2013, pp. 1-11.

[21] J. Allport, M. Jupp, A. Pezouvanis, G. Janicki, A. Pieronczyk, A. Day, P. Olley, B. Mason, M. Ebrahimi, Turbocharger blade vibration: Measurement and validation through laser tip-timing, in: Proceedings of the 10th International Conference on Turbochargers and Turbocharging, 2012, pp. 1-10.

[22] S. Heath, M. Imregun, An improved single-parameter tip-timing method for turbomachinery blade vibration measurements using optical laser probes, International Journal of Mechanical Sciences 38 (1996) 1047-1058. doi:10.1016/0020-7403(95)00116-6.

[23] C. Lawson, P. Jvey, Turbomachinery blade vibration amplitude measurement through tip timing with capacitance tip clearance probes, Sensors and Actuators A: Physical 118 (2005) 14-24.

[24] S. Zucca, B. Epureanub, Identificazione di palette criccate in dischi di turbomacchine, (damaged blades detection in turbomachinery disks), in: Proceedings of the 43th Convegno Nazionale AIAS, 2014, pp. 1-14.

[25] P. Castellinia, N. Paone, Development of the tracking laser vibrometer: Performance and uncertainty analysis, Review of Scientific Instruments 71 (2000) 4639-4647. doi:10.1063/1.1319862.

[26] D. D. Maio, D. Ewins, Applications of continuous tracking sldv measurement methods to axially symmetric rrotating structures using different excitation methods, MechanicalSystemsandSignalProcessing 24 (2010) 3013-3036.

[27] A. K. Reinhardt, J. R. Kadambi, R. D. Quinn, Laser vibrometry measurements of rotating blade vibrations, Journal of Engineering for Gas Turbines and Power 117 (2007) 484-488.

[28] R. A. Cookson, P. Bandyopadhyay, A fiber-optic laser-doppler probe for vibration analysis of rotating machines, Journal of Engineering for Gas Turbines and Power 102 (2009) 607-612.

[29] Y. Hirata, Non-harmonic fourier analysis available for detecting very low-frequency components, Journal of Sound and Vibration 287 (2005) 611-613.

[30] T. Yoshizawa, S. Hirobayashi, T. Misawa, Noise reduction for periodic signals using high resolution frequency analysis, EURASIP Journal of Audio, Speech, and Music Processing 2011 (2011) 5-24.

[31] A. Oberholster, P. Heyns, A study of the non-harmonic fourier analysis technique, in: Proceedings of the 21st International Congress on Condition Monitoring and Diagnostic Engineering Management, 2009, pp. 1-9.

[32] A. Oberholster, P. Heyns, Eulerian laser doppler vibrometry: Online blade damage identification on a multi-blade test rotor, Mecha 25 (2011) 344-359.

[33] P.Neri, B.Peeters, Non-harmonic fourier analysis for bladed wheels damage detection, Journal of Sound and Vibration 356 (2015) 181-194. doi:10.1016/j.jsv.2015.06.048. 


\section{List of Figures}

1 Flux diagram of the NHFA algorithms for damage detection: (a) previous algorithm and (b)

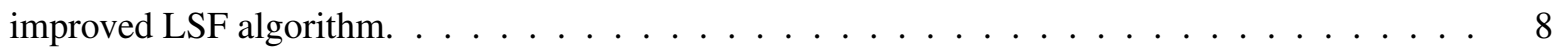

2 Finite element model scheme: blade with a growing crack. . . . . . . . . . . . . 9

3 Improved algorithm results: (a) no filtering applied to the signal and (b) moving average filter

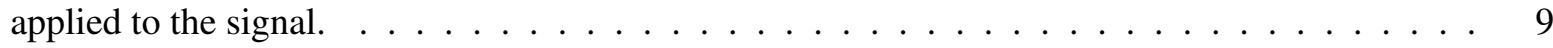

4 Frequency estimation with the previous algorithm for comparison with Fig.3(b) . . . . . . . 10

5 Estimated frequency using the improved algorithm: zoomed view for early stage damage detection. 11 